\title{
Robust approach to monitoring Lagrangian transport in very large volumes
}

\author{
F. Kaiser*, A. Haramis, J. Galler, D. E. Rival \\ Department of Mechanical and Materials Engineering, Queen's University, Kingston, Ontario, Canada \\ * f.kaiser@queensu.ca
}

\begin{abstract}
State-of-the-art flow measurements utilize four or more high-speed cameras to perform highly-accurate Lagrangian particle tracking (LPT) in small to medium-sized measurement volumes (Schanz et al., 2016). Hou et al. (2021) suggested a novel approach to allow measurements in significantly larger measurement volumes $\left(O\left(10 \mathrm{~m}^{3}\right)\right)$ while reducing the experimental effort. A single camera is used to track centimeter-sized soap bubbles in three dimensions by not only evaluating the bubble-center location but also the bubbleimage size. Possible applications of the suggested approach include - but are not limited to - measurements in industrial wind tunnels (Hou et al., 2021), full-scale measurements in the atmospheric boundary layer (Rosi et al., 2014; Toloui et al., 2014), and the characterization of airflow in indoor spaces, such as offices or classrooms (Kähler et al., 2020). In the context of the recent pandemic, the latter application could help to reduce infection risk by designing appropriate air circulation. Hereby, frequent air exchange is recommended, while direct airflow from individual to individual should be avoided (WHO, 2020). The present study strives to optimize and simplify the experimental set-up as well as to characterize the accuracy of the novel single-camera approach. Figure 11a) shows the set-up used to characterize the novel approach.
\end{abstract}

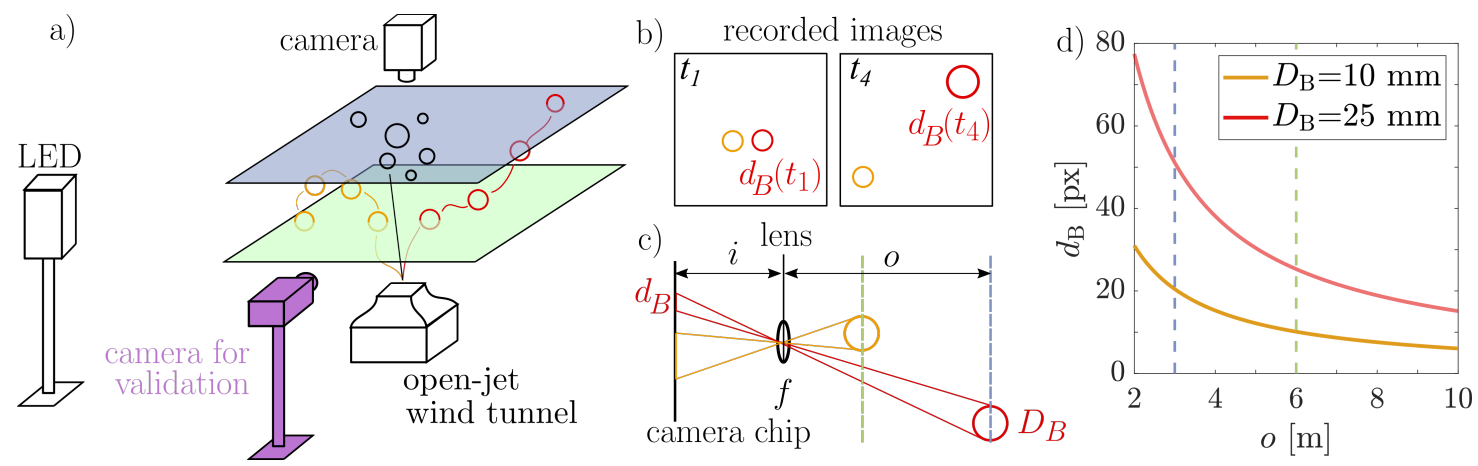

Figure 1: (a) Sketch of the experimental set-up; (b) bubble images recorded at $t_{1}$ and $t_{4}$ with varying bubbleimage size $\left(d_{b}\right)$; (c) linear optics producing the bubble images of size $d_{B}$ dependent on bubble size $\left(D_{B}\right)$ and position $(o)$; and (d) bubble-image size $\left(d_{B}\right)$ in pixels as a function of object distance $o$ with a $f=35 \mathrm{~mm}$ lens and a pixel size of $10 \mu \mathrm{m}$. Dashed lines visualize the planes shown in (a).

The flow over an open-jet wind-tunnel nozzle (cross section $0.13 \mathrm{~m} \times 0.13 \mathrm{~m}$ ) was captured in a $1 \mathrm{~m} \times 1 \mathrm{~m}$ $\times 1.2 \mathrm{~m}$ measurement volume via two cameras: (i) a main camera that was mounted above the wind tunnel; and (ii) a secondary camera that was used for validation purposes. Large soap bubbles $\left(D_{B} \approx 30 \mathrm{~mm}\right)$ are illuminated by a light source placed perpendicular to both cameras. Dependent on the distance of the bubble to the camera $o(t)$ the bubble-image size $d_{B}(t)$ varies in time; see figure 11(b). Assuming $o \gg i$ (image distance $i$, see figure 1 ; ) the lens equation leads to $i \approx f$, which in turn simplifies the magnification equation to

$$
o \approx \frac{D_{\mathrm{B}} f}{d_{\mathrm{B}}} .
$$

Using equation 1 , the object distance $o$ and thus the three-dimensional position of a bubble can be determined with a single camera if $D_{B}$ is known. Figure 1 (d) presents the interplay of $D_{B}, o$ and $d_{B}$ for two different 
bubble sizes. To simplify future experiments, a custom bubble generator has been developed to produce uniformly-sized bubbles $\left(D_{B} \approx 30 \mathrm{~mm}\right)$. Note that an error of $D_{B}$ propagates linearly to the estimated object distance (see equation (1) and that therefore a high degree of bubble-size uniformity is required. The bubble generator is designed such that it can be placed in a uniform flow and produce bubbles without an additional air supply. The uniformity of the generated bubbles was evaluated by placing the bubble generator on the open-jet wind tunnel and $D_{B}$ could be measured via shadowgraphy. The resulting probability distribution is presented in figure 2(a). For the present bubble-generator design $97 \%$ of all measured bubbles were within $\pm 5 \%$ of the mean diameter.

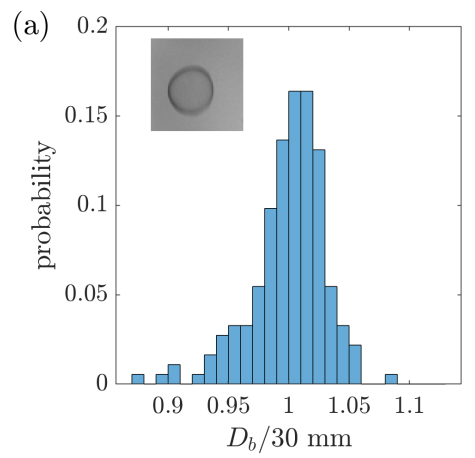

(b)

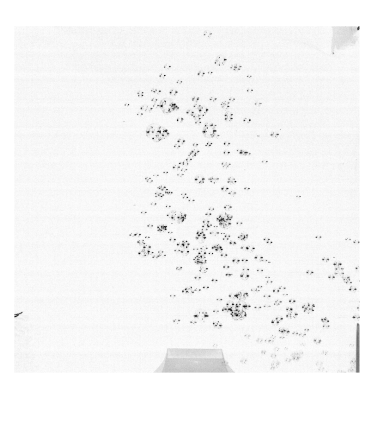

(c)

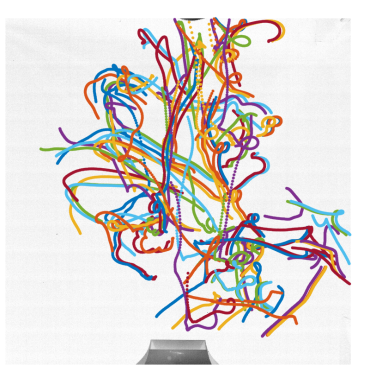

Figure 2: (a) Bubble-size probability distribution of the novel bubble generator (sample size: 180 bubbles). The inset shows an exemplary bubble image generated via shadowgraphy. (b) Raw image of bubbles and their glare points. (c) Exemplary tracks of selected bubbles. Two glare points per bubble result in pairs of similar tracks.

An additional unknown for the present single-camera approach are the errors made while determining the bubble-image size $d_{B}$. In the present set-up the light source is placed perpendicular to the camera(s). As such the bubble image consists of two glare points with a glare-point spacing $d_{G}=\sqrt{2} / 2 d_{B}$; see figure 2 (b). Classical PIV and PTV utilize Gaussian peak fitting to approximate particle position at $0.1 \mathrm{px}$ accuracy. However, as the bubble glare points have a non-Gaussian brightness distribution, larger errors are expected. To quantify these errors that are introduced by falsely estimating $d_{B}$, a starting vortex generated by the openjet wind tunnel is captured via two cameras (figure 2k). By recording the same bubble from two different perspectives (black and purple cameras in figure 1a), the bubble positions can be determined in two ways: (i) by using two perspectives and photogrammetry; and (b) equation (1). The results of both approaches are compared and provide an estimate of the accuracy of the single-camera approach first suggested in $\mathrm{Hou}$ et al. (2021).

\section{References}

Hou J, Kaiser F, Sciacchitano A, and Rival DE (2021) A novel single-camera approach to large-scale, threedimensional particle tracking based on glare-point spacing. Experiments in Fluids 62:100

Kähler CJ, Fuchs T, Mutsch B, and Hain R (2020) School education during the sars-cov-2 pandemic-which concept is safe, feasible and environmentally sound?. medRxiv

Rosi GA, Sherry M, Kinzel M, and Rival DE (2014) Characterizing the lower log region of the atmospheric surface layer via large-scale particle tracking velocimetry. Exp Fluids 55:1736

Schanz D, Gesemann S, and Schröder A (2016) Shake-the-box: Lagrangian particle tracking at high particle image densities. Experiments in Fluids 57:70

Toloui M, Riley S, Hong J, Howard K, Chamorro LP, Guala M, and Tucker J (2014) Measurement of atmospheric boundary layer based on super-large-scale particle image velocimetry using natural snowfall. Exp Fluids 55:1737

WHO (2020) Q\&A: ventilation and air conditioning in public spaces and buildings and COVID-19 Available at: www.who.int . Last accessed: 01/13/2021 